\title{
ARTí́cUlO
}

\section{Amplificación del gen $r b c L$ revela primer registro de Porphyra mumfordii (Bangiales, Rhodophyta) en la bahía de Valparaíso, Chile central}

$r b c L$ gene amplification reveals first record of Porphyra mumfordii (Bangiales, Rhodophyta) in Valparaíso Bay, central Chile

\author{
Pilar Muñoz-Muga ${ }^{*}$, Héctor Romo², Carlos Calderón ${ }^{3}$, Oscar Evrard ${ }^{3}$ y Humberto Díaz ${ }^{1,3}$ \\ ${ }^{1}$ Facultad de Ciencias del Mar y de Recursos Naturales, Universidad de Valparaíso, Avenida Borgoño 16344, Viña del Mar, Chile. \\ *pilar.munoz@uv.cl. \\ ${ }^{2}$ Facultad de Ecología y Recursos Naturales, Sede Viña del Mar, Universidad Andrés Bello, Calle Quillota 980, Viña del Mar, Chile \\ ${ }^{3}$ Laboratorio de Biotecnología, Biotecnos S.A., Avenida Brasil 2104, Valparaíso, Chile
}

\begin{abstract}
The genus Porphyra, commonly found along Chilean coast, is an important commercial algal food known as nori. There are some specimens of Porphyra found at Montemar Marine Protected Area ( $32^{\circ} 57^{\prime} \mathrm{S}$; $71^{\circ} 33^{\prime} \mathrm{W}$ ) in Valparaíso Bay, which taxonomical identity is not clear. This study aimed to identify long-bladed specimens of Porphyra inhabiting the upper, mid and lower intertidal in Valparaíso Bay. To do so, 30 thalli of Porphyra were collected during the winter of 2015 in Montemar. All samples were subjected to $r b c \mathrm{~L}$ molecular marker amplification. However, only 18 individuals were sequenced successfully. In addition, a morphological description was conducted. The $r b c \mathrm{~L}$ analysis showed that the longbladed specimens inhabiting Valparaíso Bay correspond to Porphyra mumfordii. Differences in size and color of thalli found on each intertidal level were recorded. This research constitutes the first record of $P$. mumfordii for central Chile.
\end{abstract}

Key words: nori, Porphyra, rbcL, Southeast Pacific

Resumen.- El género Porphyra, comúnmente encontrado a lo largo de las costas chilenas, es un importante alimento algal conocido como nori. Existen representantes de Porphyra del Área Marina Protegida Montemar ( $32^{\circ} 57^{\prime} \mathrm{S}$; $\left.71^{\circ} 33^{\prime} \mathrm{W}\right)$ en la bahía de Valparaíso, cuya identidad taxonómica no está clara. Este estudio tuvo como propósito identificar los ejemplares de fronda alargada de Porphyra, que habitan el intermareal superior, medio e inferior de la bahía de Valparaíso. Para llevar a cabo este objetivo, se recolectaron 30 talos de Porphyra durante el invierno de 2015 en Montemar. En todas las muestras se amplificó el marcador molecular $r b c \mathrm{~L}$, sin embargo solo en 18 individuos se logró un secuenciamiento exitoso. Además, se llevó a cabo una descripción morfológica. El análisis de las secuencias del gen rbcL mostró que los especímenes de fronda alargada que habitan el intermareal de la bahía de Valparaíso corresponden a Porphyra mumfordii. Se presentaron diferencias en el tamaño y coloración de los talos provenientes de distintos niveles del intermareal. Esta investigación constituye el primer registro de P. mumfordii para Chile central.

Palabras clave: nori, Porphyra, rbcL, Pacífico sudeste

\section{INTRODUCCIÓN}

La producción de macroalgas representa una exitosa industria a nivel mundial, con un valor total estimado de 10 mil millones de dólares por año (Bixler \& Porse 2011). La mayoría de la cosecha anual ( 99\%) depende de sólo 10 países. Chile ocupa el séptimo lugar, ya que registra un promedio de $430.000 \mathrm{t}$ de algas desembarcadas en la última década (FAO 2016). Cinco géneros (Saccharina, Undaria, Porphyra, Eucheuma/Kappaphycus, Gracilaria) representan el 98\% de los cultivos algales (Yarish \& Pereira 2008). De estos, el más rentable corresponde a Porphyra, conocida internacionalmente como nori, debido a su alto valor de mercado (McHugh 2003). En Chile este recurso recibe el nombre de luche y se extraen cerca de $100 \mathrm{t}$ al año, biomasa que proviene exclusivamente de las praderas naturales (SERNAPESCA 2016).

Porphyra C.Agardh es un género cosmopolita de algas rojas (Bangiales, Rhodophyta) que crece desde la parte alta del intermareal hasta el submareal somero, en las costas de aguas temperadas, polares y tropicales. Su reproducción involucra una alternancia heteromórfica de generaciones, con un gametofito folioso de marcada 
estacionalidad y un esporofito, que lleva por nombre fase conchocelis, de vida perenne (Graham et al. 2009).

Tradicionalmente se utilizaban en la clasificación de Porphyra caracteres morfológicos (e.g., Kurogi 1972). Sin embargo, la presencia de especies crípticas y morfologías similares hacen que el uso de estos caracteres por si solos produzca resultados confusos (Brodie \& Irvine 1997, Broom et al. 2002, Neefus et al. 2002, Broom et al. 2004, Lindstrom 2008).

Así, la utilización de secuencias nucleotídicas se ha transformado en un aspecto indispensable cuando se identifican especies de Porphyra (Lindstrom \& Fredericq 2003). El marcador molecular cloroplastidial $r b c L$ (gen de la subunidad grande de la ribulosa bifosfato carboxilasa, por sus siglas en inglés) ha sido ampliamente usado en Bangiales, logrando impulsar notables cambios en la taxonomía de este grupo (e.g., Lindstrom \& Fredericq 2003, Lindstrom 2008, Broom et al. 2010, Sutherland et al. 2011, Mateo-Cid et al. 2012, Mols-Mortensen et al. 2012, Deng et al. 2014, Mols-Mortensen et al. 2014, Lindstrom et al. 2015).

Aun cuando hay 186 especies registradas a nivel mundial, sólo 57 presentan actualmente un estatus taxonómico válido (Guiry \& Guiry 2018). En la bahía de Valparaíso, ubicada en Chile central, se han registrado 2 especies de Porphyra: Porphyra umbilicalis (Linnaeus) J.Agardh y Porphyra columbina Motagne 1845 (Ramírez \& Santelices 1991), ahora Pyropia orbicularis M.E.Ramírez, L.Contreras Porcia \& M.-L.Guillemin (Ramírez et al. 2014). De forma posterior, González \& Santelices (2003) describieron una mayor diversidad específica para la zona, sumando en carácter preliminar mediante observaciones morfológicas a Porphyra woolhouseae Harvey, Porphyra miniata (C.Agardh) C.Agardh (ahora Wildemania miniata (C.Agardh) Foslie), Porphyra lanceolata (Setchell \& Hus) G.M.Smith (ahora Pyropia lanceolata (Setchell \& Hus) S.C.Lindstrom), Porphyra torta V.Krishnamurthy (ahora Pyropia torta (V.Krishnamurthy) S.C.Lindstrom), Porphyra thuretii Setchell \& E.Y.Dawson (ahora Pyropia thuretii (Setchell \& E.Y.Dawson) J.E.Sutherland, L.E.Aguilar-Rosas \& R.Aguilar-Rosas), Porphyra capensis Kützing, Porphyra pseudolinearis Ueda (ahora Pyropia pseudolinearis (Ueda) N.Kikuchi, M.Miyata, M.S.Hwang \& H.G.Choi) y Porphyra linearis Greville.

Al realizar visitas reiteradas a la zona rocosa del Área Marina Protegida Montemar en la bahía de Valparaíso, se ha observado la existencia de grupos de individuos de
Porphyra con frondas alargadas de distintos tamaños. Estos se encuentran distribuidos en la zona superior, media e inferior del intermareal, y sus características morfológicas no coinciden precisamente con ninguna de las especies antes descritas de forma oficial para Chile central.

Debido a la potencial expansión del comercio de Porphyra en Chile y tomando en cuenta el impulso que el Gobierno de Chile le quiere dar al cultivo de algas en pequeña escala, reflejado en el Proyecto de Ley para el cultivo y repoblamiento de algas (Boletín 9151-21) ${ }^{1}$, es de vital importancia contribuir al conocimiento de la diversidad de Bangiales en las costas de Chile. En este contexto, el presente trabajo se centra en identificar taxonómicamente los ejemplares de fronda alargada de Porphyra que habitan el intermareal superior, medio e inferior de la bahía de Valparaíso, a través de la aplicación de secuenciamiento del gen $r b c \mathrm{~L}$ y el análisis de aspectos morfológicos.

\section{MATERIALES Y MÉTODOS}

El sitio de estudio correspondió al Área Marina Protegida Montemar (3257’s; 71³3’W) en la bahía de Valparaíso, Región de Valparaíso, Chile central (Fig. 1). Durante el invierno austral (24 junio 2015) se distinguieron las agregaciones de talos de Porphyra del intermareal rocoso superior, medio e inferior de esta localidad (Fig. 2). Se procedió a colocar un cuadrante (área $=0,021 \mathrm{~m}^{2}$ ) de forma aleatoria sobre los talos y se recolectaron todos los individuos a mano, cuidando de extraerlos completos. Parte del material se reservó para montarlo en papel de herbario sin formalina (Ramírez 1995), el que sirvió para realizar observaciones morfológicas. El resto de los especímenes obtenidos se transportaron al Laboratorio de Biotecnología de la empresa Biotecnos S.A. en Viña del Mar (Región de Valparaíso), donde fueron lavados con agua destilada y almacenados a $-20^{\circ} \mathrm{C}$ en tubos Eppendorf por separado ( $n=10$ para cada uno de los niveles intermareales).

\footnotetext{
${ }^{1}$ Boletín 9151-21. Subsecretaría de Pesca y Acuicultura de Chile. Proyecto Ley para el cultivo y repoblamiento de algas. 222 Legislatura 361. <https://www.camara.cl/pley/ pley_detalle.aspx?prmID=9647\&prmBL=9151-21>
} 


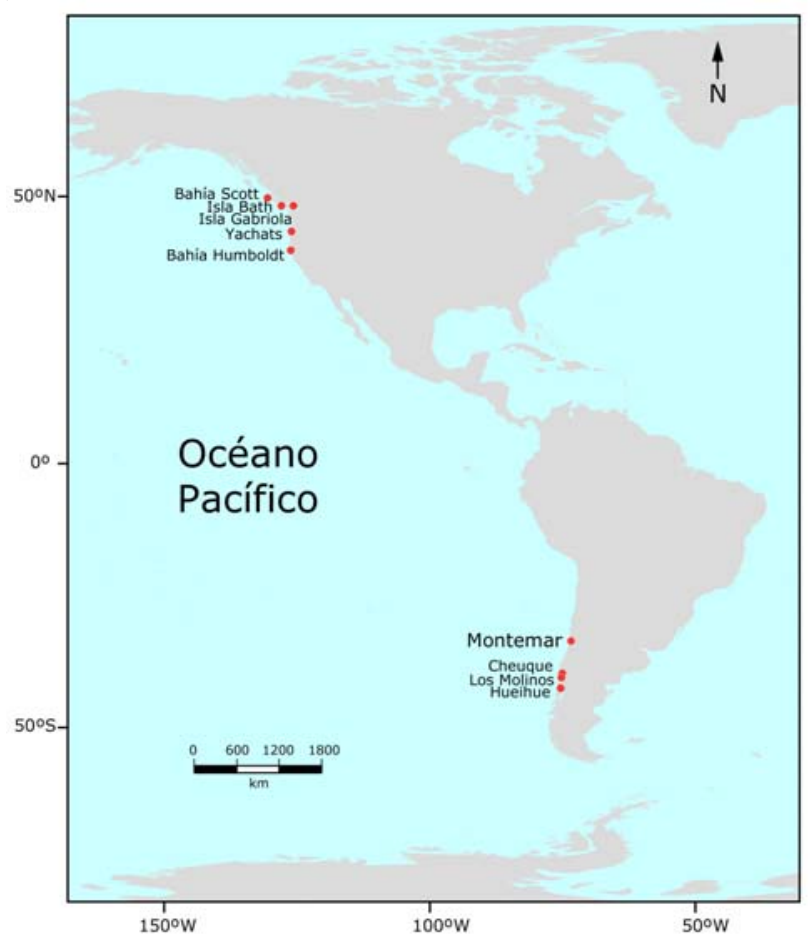

Figura 1. Mapa del Océano Pacífico Oriental indicando el sitio de muestreo Montemar en bahía de Valparaíso, además de las localidades en Canadá, EEUU y Chile donde se ha registrado previamente $P$. mumfordii (Lindstrom \& Cole 1992, Lindstrom \& Fredericq 2003, Guillemin et al. 2016) / East Pacific Ocean map indicating study site Montemar in Valparaíso Bay, in addition to the localities in Canada, USA and Chile where P. mumfordii has been previously registered are also displayed (Lindstrom \& Cole 1992, Lindstrom \& Fredericq 2003, Guillemin et al. 2016)

Posteriormente, las muestras congeladas fueron molidas con un hisopo plástico. La extracción de ADN se realizó mediante DNeasy Blood \& Tissue Kit (Qiagen, California, USA), siguiendo el protocolo incorporado para tejido animal (Spin-Column Protocol). La concentración de ADN se estimó al determinar la absorbancia a $260 \mathrm{~nm}$ en un espectrofotómetro UV/VIS Optizen Pop (Mecasys Co. Ltd, Korea) y su integridad fue medida utilizando la relación de absorbancias 260/280 nm. Las muestras con relaciones $>1,8$ se consideraron íntegras y aptas para PCR. El gen $r b c \mathrm{~L}$ fue amplificado usando los partidores F-rbcL (5' - TTG CAT AYG ATA TTG ATY TAT TTG AA -3') y RrbcS (5'- RAG CTG TTT KTA AAG GWC CAC AA -3'), descritos por Ramírez et al. (2014).

Las amplificaciones se realizaron en un termociclador Maxygen (Axygen, California, USA). La correcta amplificación se verificó realizando una electroforesis gel
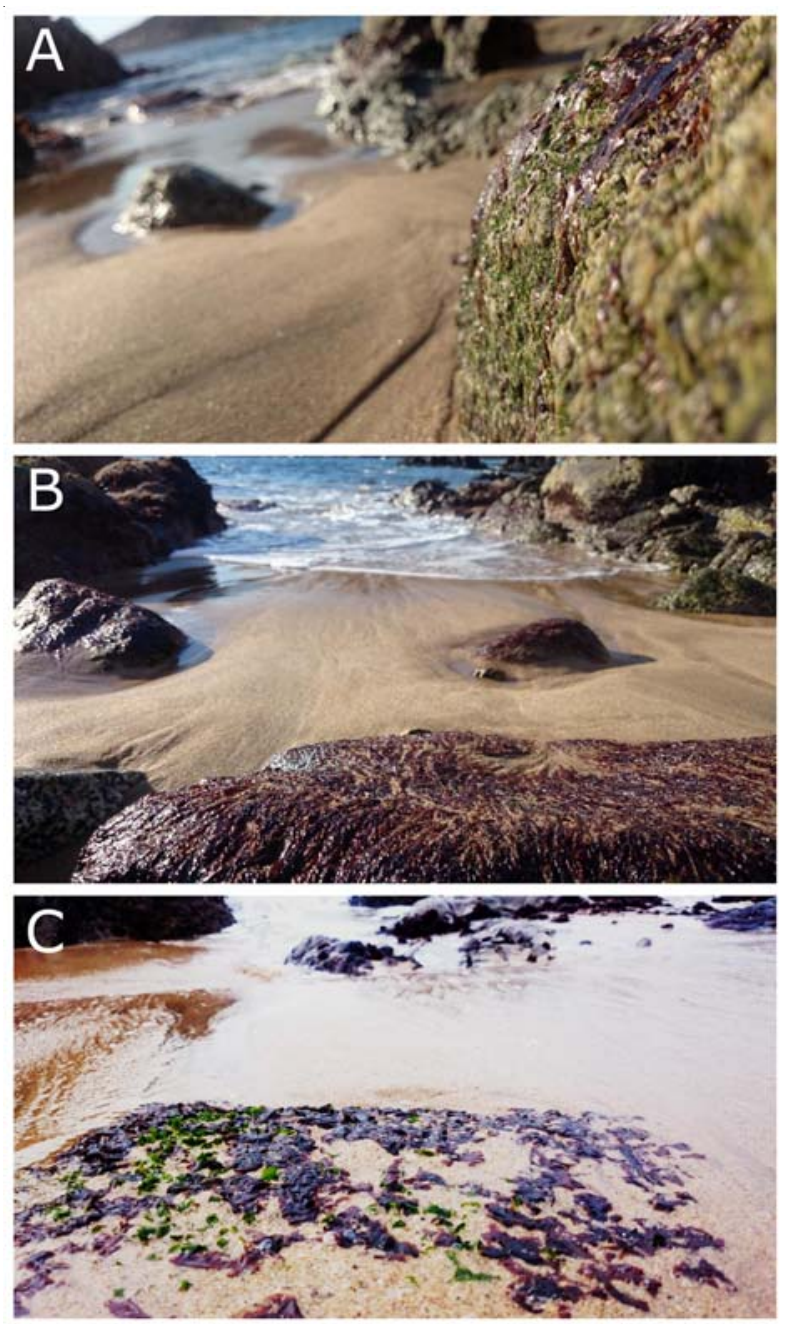

Figura 2. Frondas de Porphyra mumfordii en el intermareal superior (A), medio (B) e inferior (C) de Montemar, bahía de Valparaíso, Chile / Porphyra mumfordii thalli found on upper (A), mid (B) and lower (C) intertidal from Montemar, Valparaíso Bay, Chile

de agarosa al 1\% en tampón TBE. El gel fue marcado con la tinción GelRed ${ }^{\circledR}$. Los productos de PCR fueron purificados usando Wizard ${ }^{\circledR}$ SV Gel and PCR Clean-Up System (Promega, Madison, USA), de acuerdo a las instrucciones del fabricante.

Para la secuenciación, las muestras fueron enviadas a Macrogen Inc (Seul, Corea del Sur). Se obtuvieron secuencias de $r b c \mathrm{~L}$ para un total de 18 ejemplares de Porphyra, que fueron ingresadas a GenBank (número de acceso GenBank: KU375194 al KU375196). Se utilizó la herramienta BLAST para realizar una identificación preliminar. 
Tabla 1. Información de recolección de especímenes, número de acceso a GenBank y referencia de las secuencias de $r b c L$ utilizadas en los análisis / Specimen collection information, GenBank accession number and reference of $r b c L$ sequences used in the analyses

\begin{tabular}{|c|c|c|c|}
\hline Taxón & Localidad de recolección & $\begin{array}{l}\mathrm{N}^{\circ} \text { de acceso } \\
\text { GenBank }\end{array}$ & Referencia \\
\hline Porphyra dioica J.Brodie \& L.M.Irvine & Molin, Islas Feroe & JN703282 & Mols-Mortensen et al. 2012 \\
\hline Porphyra dioica J.Brodie \& L.M.Irvine & Krossavik, Islandia & JN787102 & Mols-Mortensen et al. 2012 \\
\hline Porphyra dioica J.Brodie \& L.M.Irvine & Gales, Reino Unido & AF081291 & Klein et al. 2003 \\
\hline Porphyra linearis Greville & Hofnin, Islandia & JN787103 & Mols-Mortensen et al. 2012 \\
\hline Porphyra linearis Greville & Maine, EEUU & JN028945 & Kucera \& Saunders 2012 \\
\hline Porphyra linearis Greville & Aberystwyth, Reino Unido & HQ687547 & Sutherland et al. 2011 \\
\hline Porphyra mumfordii S.C.Lindstrom \& K.M.Cole & $\begin{array}{l}\text { Intermareal alto, Montemar, } \\
\text { Chile }\end{array}$ & KU375194 & Este estudio \\
\hline Porphyra mumfordii S.C.Lindstrom \& K.M.Cole & $\begin{array}{l}\text { Intermareal medio, Montemar, } \\
\text { Chile }\end{array}$ & KU375195 & Este estudio \\
\hline Porphyra mumfordii S.C.Lindstrom \& K.M.Cole & $\begin{array}{l}\text { Intermareal bajo, Montemar, } \\
\text { Chile }\end{array}$ & KU375196 & Este estudio \\
\hline Porphyra mumfordii S.C.Lindstrom \& K.M.Cole & Columbia Británica, Canadá & KP904092 & Lindstrom et al. 2015 \\
\hline Porphyra mumfordii S.C.Lindstrom \& K.M.Cole & California, EEUU & KP904078 & Lindstrom et al. 2015 \\
\hline Porphyra mumfordii S.C.Lindstrom \& K.M.Cole & Oregón, EEUU & EU223113 & Lindstrom 2008 \\
\hline Porphyra mumfordii S.C.Lindstrom \& K.M.Cole & Washington, EEUU & EU223111 & Lindstrom 2008 \\
\hline Porphyra mumfordii S.C.Lindstrom \& K.M.Cole & Los Molinos, Chile & KP781809 & Guillemin et al. 2016 \\
\hline Porphyra mumfordii S.C.Lindstrom \& K.M.Cole & Cheuque, Chile & KP781768 & Guillemin et al. 2016 \\
\hline Porphyra purpurea (Roth) C.Agardh & New Brunswick, Canadá & JN028949 & Kucera \& Saunders 2012 \\
\hline Porphyra purpurea (Roth) C.Agardh & Qaqortoq, Groenlandia & KF478754 & Mols-Mortensen et al. 2014 \\
\hline Porphyra purpurea (Roth) C.Agardh & Litstock, Reino Unido & HQ687516 & Sutherland et al. 2011 \\
\hline Porphyra umbilicalis Kützing & Terranova y Labrador, Canadá & JN028956 & Kucera \& Saunders 2012 \\
\hline Porphyra umbilicalis Kützing & Hirsholmene, Dinamarca & JN847248 & Mols-Mortensen et al. 2012 \\
\hline Porphyra umbilicalis Kützing & Nuuk, Groenlandia & KF478755 & Mols-Mortensen et al. 2014 \\
\hline Porphyra umbilicalis Kützing & Hellnar, Islandia & JN787114 & Mols-Mortensen et al. 2012 \\
\hline Porphyra umbilicalis Kützing & Maine, EEUU & JN028957 & Kucera \& Saunders 2012 \\
\hline Porphyra umbilicalis Kützing & Skorpa, Noruega & JN847247 & Mols-Mortensen et al. 2012 \\
\hline Porphyra umbilicalis Kützing & Sidmouth, Reino Unido & HQ687559 & Sutherland et al. 2011 \\
\hline $\begin{array}{l}\text { Pyropia orbicularis M.E.Ramírez, L.Contreras } \\
\text { Porcia \& M.-L.Guillemin }\end{array}$ & Maitencillo, Chile & KF479481 & Ramírez et al. 2014 \\
\hline $\begin{array}{l}\text { Pyropia orbicularis M.E.Ramírez, L.Contreras } \\
\text { Porcia \& M.-L.Guillemin }\end{array}$ & Maitencillo, Chile & KF479483 & Ramírez et al. 2014 \\
\hline $\begin{array}{l}\text { Pyropia orbicularis M.E.Ramírez, L.Contreras } \\
\text { Porcia \& M.-L.Guillemin }\end{array}$ & Maitencillo, Chile & KF479498 & Ramírez et al. 2014 \\
\hline $\begin{array}{l}\text { Pyropia tenera KAN (Kjellman) N.Kikuchi, } \\
\text { M.Miyata, M.S.Hwang \& H.G.Choi }\end{array}$ & Kanagawa, Japón & AB366148 & Niwa et al. 2009 \\
\hline $\begin{array}{l}\text { Pyropia tenera }(\text { Kjellman) N.Kikuchi, M.Miyata, } \\
\text { M.S.Hwang \& H.G.Choi }\end{array}$ & Kawaura, Japón & $\mathrm{AB} 118576$ & Kito et al. datos no publicados \\
\hline $\begin{array}{l}\text { Pyropia tenera }(\text { Kjellman) N.Kikuchi, M.Miyata, } \\
\text { M.S.Hwang \& H.G.Choi }\end{array}$ & Kunamoto, Japón & AB243206 & Niwa et al. $2008 \mathrm{~b}$ \\
\hline $\begin{array}{l}\text { Pyropia tenera }(\text { Kjellman) N.Kikuchi, M.Miyata, } \\
\text { M.S.Hwang \& H.G.Choi }\end{array}$ & Yanaguchi, Japón & AB287949 & Taruta et al. datos no publicados \\
\hline
\end{tabular}


Las secuencias obtenidas de terreno junto con otras secuencias de Porphyra y Pyropia (grupo externo: Py. orbicularis, Py. tenera) descargadas desde GenBank (Tabla 1 ), fueron editadas (largo $=446 \mathrm{pb}$ ) y posteriormente alineadas usando la función ClustalW del programa BioEdit 7.2.5 (Hall 1999). El alineamiento sugerido por el programa fue corroborado de forma manual. Mediante el programa MEGA 6.06 (<https://www.megasoftware.net>) se realizó un agrupamiento Neighbor-joining (Tamura et al. 2011). El soporte de los nodos se validó mediante una prueba bootstrap con 1.000 réplicas. Del análisis de este agrupamiento se pudo inferir la identidad taxonómica de los individuos de Porphyra. Además se calcularon estimados de diversidad inter e intra grupos (i.e., intermareal alto, medio, bajo).

El mapa del océano Pacífico fue elaborado usando la librería maptools del programa R (R Development Core Team 2014). Las figuras fueron editadas mediante los programas Adobe ${ }^{\circledR}$ Illustrator CC y Adobe ${ }^{\circledR}$ Photoshop CS3.

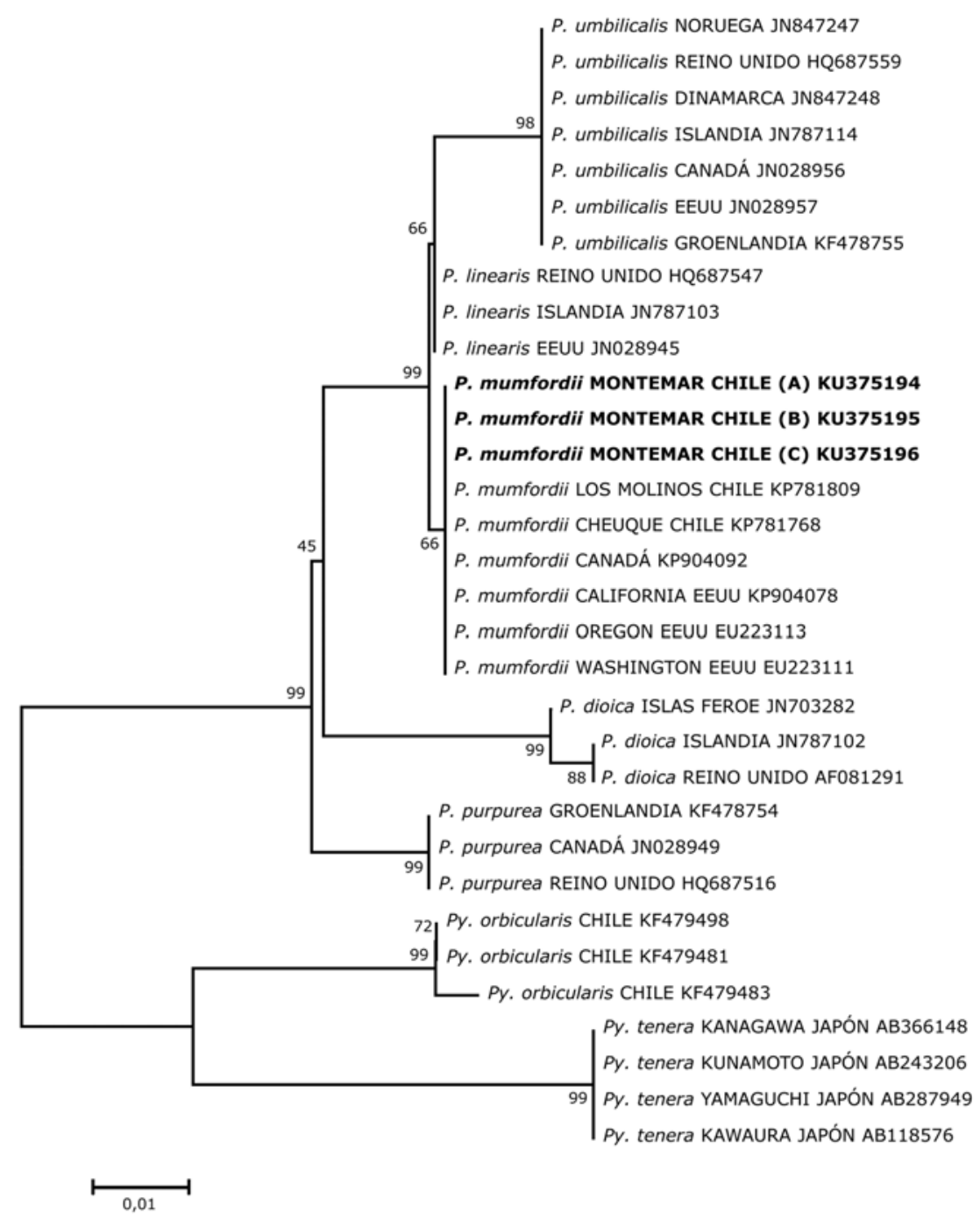

Figura 3. Agrupamiento de especies de Porphyra inferido a partir del análisis de secuencias del gen rbcL mediante la metodología Neighbor-joining. Py. tenera y Py. orbicularis fueron usados como grupo externo. Los valores de soporte (>40\%) se muestran delante de cada nodo. Los ejemplares recolectados del intermareal superior (A), medio (B) e inferior (C) de Montemar se encuentran destacados en negrita / Neighbor-joining clustering of Porphyra species infered from $r b c L$ sequences. Py. tenera and Py. orbicularis were used as outgroup. Bootstrap values (>40\%) are indicated next to branches. Specimens collected from upper (A), mid (B) and lower (C) intertidal in Montemar are in bold 


\section{Resultados}

El agrupamiento Neighbor-joining obtenido a partir de las secuencias del gen $r b c \mathrm{~L}$ (Fig. 3), sugiere que los individuos de Porphyra de fronda alargada del intermareal superior, medio e inferior de Montemar, bahía de Valparaíso, corresponden a Porphyra mumfordii S.C.Lindstrom \& K.M.Cole, cuya localidad tipo es Isla Gabriola (Fig. 1), Columbia Británica, Canadá (100\% de identidad de acuerdo a BLAST de GenBank). Todos los ejemplares de $P$. mumfordii analizados, incluidos tanto los habitantes del Pacífico Norte como Sur, forman un solo clado. Este resultado se valida por los valores de diversidad media inter e intra grupos $(0 \%$ de diferencias de bases por sitio). A nivel genérico, se observa un nodo altamente sustentado (bootstrap=99\%), que está compuesto por 5 especies de Porphyra provenientes de localidades variadas de las costas del Pacífico y Atlántico:
P. mumfordii, P. linearis, P. umbilicalis, $P$. dioica y $P$. purpurea. Por otra parte, las especies de Pyropia utilizadas en este estudio como grupo externo, forman un grupo aparte.

En cuanto a la morfología de los ejemplares de $P$. mumfordii que habitan Montemar (Fig. 4), se corroboran las diferencias en el tamaño y coloración de los talos provenientes de los distintos niveles del intermareal que se habían observado en terreno con anterioridad. Los individuos del intermareal superior son más pequeños (largo $<10 \mathrm{~cm}$; ancho $<0,5 \mathrm{~cm}$ ) y presentan una coloración más clara, en un tono café-amarillo, lo que dificulta la visualización de los márgenes femeninos y masculinos. En el intermareal medio, los talos alcanzan $30 \mathrm{~cm}$ de largo y tienen un color rojo intenso, salvo en los márgenes masculinos, los que contrastan por su aspecto
A
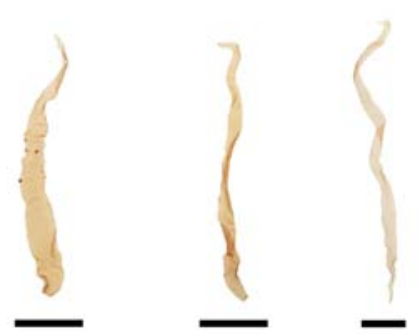

B

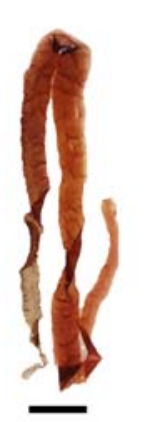

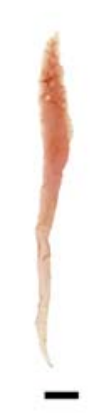

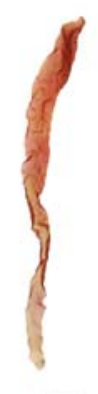

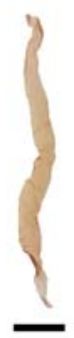
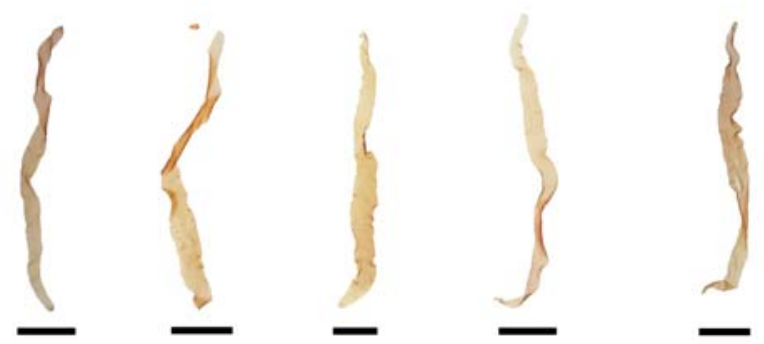

C
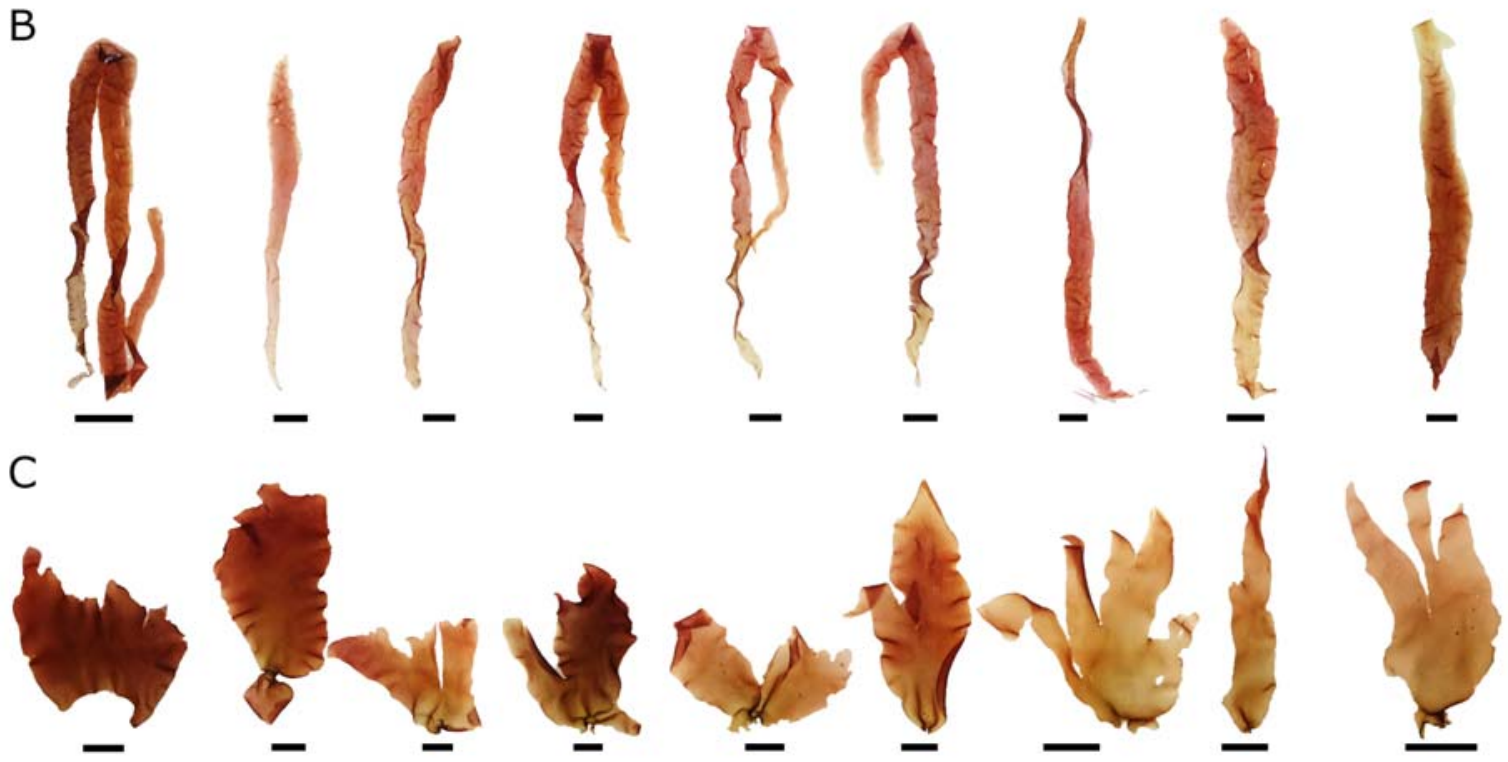

Figura 4. Hábitos del gametofito folioso de Porphyra mumfordii recolectados en el intermareal superior (A), medio (B) e inferior (C) de Montemar, bahía de Valparaíso, Chile. Barra de escala $\mathbf{1} \mathrm{cm} /$ Habits of Porphyra mumfordii foliose gametophyte collected at upper (A), mid (B) and lower (C) intertidal in Montemar, Valparaíso Bay, Chile. Scale bar $=1 \mathrm{~cm}$ 
blanquecino. Hacia el sector inferior del intermareal, las frondas destacan por su gran ancho (hasta $5 \mathrm{~cm}$ ), el que incluso puede superar el largo. El color es más variable, observándose talos de distintas tonalidades de rojo y amarillo oscuro. Cabe señalar la alta frecuencia de rasgaduras que se observa en este grupo de individuos.

\section{Discusión}

El presente trabajo representa el primer registro de Porphyra mumfordii para la bahía de Valparaíso, ampliándose de esta manera el rango de distribución de esta especie en el Pacífico Sur. La identificación taxonómica se logró mediante el análisis de las secuencias del gen $r b c \mathrm{~L}$ y la correspondencia con las descripciones morfológicas del tipo de P. mumfordii. Además se observó una gran variabilidad en el tamaño y coloración de los talos, dependiendo del nivel intermareal en que se encontraban.

De acuerdo a la revisión de Guiry \& Guiry (2018), P. mumfordii se distribuye exclusivamente en la costa Pacífica de Norteamérica (Fig. 1), i.e., Columbia Británica (Bahía Scott, Isla Gabriola, Isla Bath), Oregón (Yachats) y California (Bahía Humboldt). Si bien en una publicación de Lindstrom (2008) se menciona que se utilizaron muestras de P. mumfordii provenientes de Chile, la autora no se hace cargo de la ampliación del rango de distribución de la especie hacia el Pacífico Sur. Incluso en un trabajo posterior (Lindstrom et al. 2015) se reafirma que el límite sur de la distribución específica es California. Recientemente Guillemin et al. (2016) han identificado, mediante los marcadores $r b c \mathrm{~L}$ y COI, ejemplares de $P$. mumfordii provenientes de dos localidades del sur de Chile: Cheuque y Los Molinos (Fig. 1). Debido a que estas secuencias resultaron ser idénticas a las encontradas en el Pacífico Noreste (Tabla 1), los autores sugieren un evento de dispersión reciente, lo que podría respaldar el escenario de una introducción reciente en Chile.

Neefus et al. (2008) demostraron que el marcador molecular $r b c \mathrm{~L}$ es adecuado para resolver problemas al nivel específico en Porphyra, ya que presenta una tasa de cambio relativamente alta. La gran divergencia entre taxa, sin embargo, puede ocasionar menores valores de bootstrap en los clados más profundos (Lindstrom \& Fredericq 2003), tal como se registró en nuestro estudio. El número de especies usadas puede influir igualmente en la validez de estos análisis, al sumar más taxa se observa una mejora en el soporte de los clados y un aumento en la precisión de los modelos evolutivos (Pollock \& Bruno
2000). Lamentablemente, en este momento se encuentran pocas secuencias de especies cercanas a $P$. mumfordii en GenBank, pero se espera que a medida que éstas aumenten, con el ingreso de más especies de Porphyra, mejore a su vez la calidad de los agrupamientos. Asimismo, Sutherland et al. (2011) llaman a tener precaución con los taxa encontrados en literatura previa a la restructuración de Porphyra.

En lo que respecta a características morfológicas, la descripción del tipo (Lindstrom \& Cole 1992) detalla que P. mumfordii puede presentar un talo ovado, obovado, cuneado, orbiculado o lancelado. La coloración varía desde verde oliva a verde grisáceo, con los márgenes reproductivos en un tono púrpura-rojizo (femeninos) o blanquecino (masculino). Las frondas alcanzan hasta 45 $\mathrm{cm}$ de longitud, variando su ancho entre 0,4 y $8,4 \mathrm{~cm}$. Su sexualidad es dioica, pero también se pueden encontrar organismos monoicos. Esta gran variabilidad intraespecífica explicaría las notorias diferencias morfológicas observadas en los grupos de ejemplares de P. mumfordii provenientes de distintos microambientes de Montemar (intermareal superior, medio e inferior), las que incluso llevaron a pensar en un comienzo que se trataban de 3 taxa distintos.

El desarrollo de las macroalgas está influído por las fluctuaciones de los parámetros ambientales y en las costas rocosas se puede observar un gradiente provocado por las oscilaciones mareales (Chappuis et al. 2014). Por lo tanto, es posible encontrar también variaciones fenotípicas de P. mumfordii a lo largo de esta zonación vertical. Los individuos que habitan hacia sectores más superiores del intermareal deben sobrellevar la disponibilidad limitada de nutrientes (Schonbeck \& Norton 1979), el estrés causado por la desecación (Schonbeck \& Norton 1980) y la mayor susceptibilidad al daño por radiación UV (Mansilla et al. 2006), explicando así el menor tamaño y pigmentación de $P$. mumfordii encontrada en esta zona. En cambio, hacia el intermareal inferior, el desarrollo de las algas se ve perjudicado al existir una mayor competencia por el sustrato (Mangialajo et al. 2012) y presión de pastoreo (Underwood \& Jernakoff 1984), lo que sería la causa de las rasgaduras y cortes que presentan estas frondas de P. mumfordii.

Al comparar las morfologías de las especies de Porphyra descritas de forma preliminar por González \& Santelices (2003) para el área de la bahía de Valparaíso (32 $57^{\prime}$ 'S; $71^{\circ} 42^{\prime} \mathrm{W}$ ) con la especie $P$. mumfordii encontrada prácticamente en la misma localidad de Montemar (32 ${ }^{\circ} 57^{\prime} \mathrm{S} ; 7^{\circ} 33^{\prime} \mathrm{W}$ ), se revela una gran similitud 
entre las figuras de ambos trabajos. Claramente, la morfología de P. pseudolinearis (fig. 1G) y P. linearis (fig. 1H) ilustradas por González \& Santelices (2003) corresponderían a las morfologías de P. mumfordii de los niveles superiores y medios de este trabajo (Figs. 4A y B). En forma similar, aunque no tan conclusivamente, se podrían homologar las figuras $1 \mathrm{E}$ ( $P$. thuretii), F ( $P$. capensis) y quizás D (P. torta), determinadas por González \& Santelices (2003), a las formas de los niveles inferiores del intermareal de P. mumfordii ( Fig. 4C). En consecuencia, la plasticidad fenotípica de esta especie podría estar agrupando una amplia diversidad de morfologías de Porphyra de la bahía de Valparaíso en una única entidad de Porphyra mumfordii, lo que merece una atención más detenida.

La aplicación incorrecta de nombres es uno de los problemas que causa más frustración en la taxonomía de Bangiales, transformando los estudios ecológicos y de fisiología comparativa de las especies en una actividad extremadamente difícil. El uso de datos de secuencias moleculares para llevar a cabo la identificación específica en Porphyra, por lo tanto, se ha convertido en una clara necesidad (Sutherland et al. 2011).

Es necesario señalar que existen limitaciones cuando se utiliza solamente un marcador molecular al realizar identificaciones específicas, ya que la detección de hibridaciones e introgresiones resulta menos confiable (Chase et al. 2005). La metodología inicial incluía la utilización de un segundo locus: COI o gen mitocondrial que codifica para la subunidad I de la citocromo oxidasa. Lamentablemente, no se pudo lograr una amplificación de calidad mediante los partidores utilizados (Saunders 2005). Es más, Guillemin et al. (2016) han señalado que es difícil realizar comparaciones de especímenes de Bangiales con COI, porque existe una escasa disponibilidad de secuencias de este marcador en GenBank.

Por otra parte, la biomasa de Porphyra sensu lato posee un alto valor de mercado cuando es comercializada como nori (McHugh 2003), por lo que sería de gran interés iniciar este negocio en Chile. Como los recursos pesqueros son cada día más escasos, el desarrollo de la acuicultura en el mundo está creciendo sostenidamente, ya que constituye una alternativa sostenible (Duarte et al. 2007). Lindstrom (1993) señala que al seleccionar una cepa de Porphyra apropiada para el cultivo se debe poner énfasis en ejemplares con fronda larga y angosta, que posean una maduración tardía y presenten monosporas como parte de su ciclo de vida. De la misma forma se deben considerar las propiedades organolépticas, debido a los estrictos requerimientos del mercado del nori (Niwa et al. 2008a). $P$. mumfordii cuenta efectivamente con frondas de tamaño adecuado y Kusumo (1993) describió que su concentración de aminoácidos en peso seco $(37,78 \%)$ es mayor que el $30 \%$ de Pyropia yezoensis (Ji et al. 1981) y el 35,6\% de Pyropia tenera (Arasaki \& Arasaki 1986). Este conocimiento puede facilitar la selección de especies que ya se encuentran habitando la bahía de Valparaíso para la producción de nori, en vez de utilizar especies introducidas (Kucera \& Saunders 2012).

Se concluye que el análisis de las secuencias del gen $r b c \mathrm{~L}$ y las observaciones morfológicas permitieron clarificar el estatus taxonómico de los ejemplares de fronda alargada de Porphyra, que habitan el intermareal superior, medio e inferior de la bahía de Valparaíso. Se identificó a P. mumfordii, una especie que presenta alta variabilidad en su tamaño y coloración, además que nunca había sido registrada para la región. Sin embargo, es necesario el uso de un gen independiente para validar esta identificación específica. De acuerdo a los antecedentes recopilados, $P$. mumfordii tiene el potencial de convertirse en un nuevo recurso algal chileno e incorporado en la producción de nori. Se recomienda ampliar el conocimiento de esta especie, con la realización de estudios que se enfoquen en comprender su fisiología, reproducción y estacionalidad.

\section{Agradecimientos}

Esta investigación fue financiada por Biotecnos S.A. Agradecemos la labor de los cuatro evaluadores anónimos por sus comentarios y sugerencias que ayudaron a mejorar el manuscrito.

\section{LITERATURA CITADA}

Arasaki S \& T Arasaki. 1986. Lipid and fatty acid composition in the red alga Porphyra yezoensis. Japanese Journal of Phycology 34: 94-100.

Bixler HJ \& H Porse. 2011. A decade of change in the seaweed hydrocolloids industry. Journal of Applied Phycology 23(3): 321-335.

Brodie J \& LM Irvine. 1997. A comparison of Porphyra dioica sp. nov. and P. purpurea (Roth) C. Ag. (Rhodophyta: Bangiophycidae) in Europe. Cryptogamie Algologie 18: 283297.

Broom JE, WA Nelson, C Yarish, WA Jones, R Aguilar Rosas \& LE Aguilar Rosas. 2002. A reassessment of the taxonomic status of Porphyra suborbiculata, Porphyra carolinensis and Porphyra lilliputiana (Bangiales, Rhodophyta) based on molecular and morphological data. European Journal of Phycology 37: 227-235. 
Broom JES, TJ Farr \& WA Nelson. 2004. Phylogeny of the Bangia flora of New Zealand suggests a southern origin for Porphyra and Bangia (Bangiales, Rhodophyta). Molecular Phylogenetics and Evolution 31: 1197-1207.

Broom JES, WA Nelson, TJ Farr, LE Phillips \& M Clayton. 2010. Relationships of the Porphyra (Bangiales, Rhodophyta) flora of the Falkland Islands: a molecular survey using $r b c \mathrm{~L}$ and $\mathrm{nSSU}$ sequence data. Australian Systematic Botany 23(1): 27-37.

Chappuis E, M Terradas, ME Cefalì, S Mariani \& E Ballesteros. 2014. Vertical zonation is the main distribution pattern of littoral assemblages on rocky shores at a regional scale. Estuarine, Coastal and Shelf Science 147: 113-122.

Chase MW, N Salamin, M Wilkinson, JM Dunwell, RP Kesanakurthi \& N Haidar. 2005. Land plants and DNA barcodes: short-term and long-term goals. Philosophical Transactions of the Royal Society of London B: Biological Sciences 360: 1889-1895.

Deng Y, Q Lu, S Shen, Z Shen, C Tian \& J Zhu. 2014. Cytological observations and $r b c \mathrm{~L}$, nrSSU gene sequence analyses of filamentous Bangiales (Rhodophyta) from China. Journal of Applied Phycology 27(3): 1355-1363.

Duarte CM, N Marbá \& M Holmer. 2007. Rapid domestication of marine species. Science 316(5823): 382-383.

FAO. 2016. Anuario estadístico de pesca y acuicultura, 76 pp. Organización de las Naciones Unidas para la Alimentación y la Agricultura, Roma.

González A \& B Santelices. 2003. A re-examination of the potential use of central Chilean Porphyra (Bangiales, Rhodophyta) for human consumption. In: Chapman ARO, RJ Anderson, VJ Vreeland \& IR Davison (eds). Proceedings of the 17th International Seaweed Symposium, pp. 249255. Oxford University Press, Oxford.

Graham LE, JM Graham \& LW Wilcox. 2009. Algae, 616 pp. Benjamin Cummings (Pearson), San Francisco.

Guillemin M-L, L Contreras-Porcia, ME Ramírez, EC Macaya, CB Contador, H Woods, C Wyatt \& J Brodie. 2016. The bladed Bangiales (Rhodophyta) of the South Eastern Pacific: Molecular species delimitation reveals extensive diversity. Molecular Phylogenetics and Evolution 94: 814-826.

Guiry MD \& GM Guiry. 2018. AlgaeBase. National University of Ireland, Galway. <http://www.algaebase.org/>

Hall TA. 1999. BioEdit: a user-friendly biological sequence alignment editor and analysis program for Windows 95/98/ NT. Nucleic Acids Symposium Series 41: 95-98.

Ji MH, SZ Pu \& ZQ Niu. 1981. The variation in contents of various states of amino acids in Porphyra yezoensis. Proceedings of the International Seaweed Symposium 10: 431-435.

Klein AS, AC Mathieson, CD Neefus, DF Cain, HA Taylor, BW Teasdale, AL West, EJ Hehre, J Brodie, C Yarish \& AL Wallace. 2003. Identification of north-western Atlantic
Porphyra (Bangiaceae, Bangiales) based on sequence variation in nuclear SSU and plastid $r b c L$ genes. Phycologia 42(2): 109-122.

Kucera H \& GW Saunders. 2012. A survey of Bangiales (Rhodophyta) based on multiple molecular markers reveals cryptic diversity. Journal of Phycology 48(4): 869-882.

Kurogi M. 1972. Systematics of Porphyra in Japan. In: Abbott IA \& M Kurogi (eds). Contributions to the systematics of benthic marine algae of the North Pacific, pp. 167-191. Japanese Society of Phycology, Kobe.

Kusumo HT. 1993. Chemical composition of Porphyra spp. in British Columbia, Canada. Master Thesis, Department of Biological Sciences, Simon Fraser University, British Columbia, 206 pp.

Lindstrom SC. 1993. Inter- and intrapopulation genetic variation in species of Porphyra (Rhodophyta: Bangiales) from British Columbia and adjacent waters. Journal of Applied Phycology 5(1): 53-62.

Lindstrom SC. 2008. Cryptic diversity, biogeography and genetic variation in Northeast Pacific species of Porphyra sensu lato (Bangiales, Rhodophyta). Journal of Applied Phycology 20: 951-962.

Lindstrom SC \& KM Cole. 1992. The Porphyra lanceolata$P$. pseudolanceolata (Bangiales, Rhodophyta) complex unmasked: recognition of new species based on isozymes, morphology, chromosomes and distributions. Phycologia 31(5): 431-448.

Lindstrom SC \& S Fredericq. 2003. $r b c \mathrm{~L}$ gene sequences reveal relationships among north-east Pacific species of Porphyra (Bangiales, Rhodophyta) and a new species, $P$. aestivalis. Phycological Research 51(3): 211-224.

Lindstrom SC, JR Hughey \& LE Aguilar-Rosas. 2015. Four new species of Pyropia (Bangiales, Rhodophyta) from the west coast of North America: the Pyropia lanceolata species complex updated. PhytoKeys 52: 1-20.

Mangialajo L, M Chiantore, M-L Susini, A Meinesz, R Cattaneo-Vietti \& T Thibaut. 2012. Zonation patterns and interspecific relationships of fucoids in microtidal environments. Journal of Experimental Marine Biology and Ecology 412: 72-80.

Mansilla A, C Werlinger, M Palacios, NP Navarro \& $\mathbf{P}$ Cuadra. 2006. Effects of UVB radiation on the initial stages of growth of Gigartina skottsbergii, Sarcothalia crispata and Mazzaella laminarioides (Gigartinales, Rhodophyta). Journal of Applied Phycology 18(3-5): 451-459.

Mateo-Cid LE, AC Mendoza-González, J Díaz, A Sentíes, FF Pedroche, J Diego \& S Heredia. 2012. A new species of Pyropia (Rhodophyta, Bangiaceae), from the Pacific coast of Mexico, based on morphological and molecular evidence. Phytotaxa 54: 1-12.

McHugh DJ. 2003. A guide to the seaweed industry, 118 pp. Food and Agriculture Organization of the United Nations, Rome. 
Mols-Mortensen A, CD Neefus, R Nielsen, K Gunnarsson, S Egilsdóttir, PM Pedersen \& J Brodie. 2012. New insights into the biodiversity and generic relationships of foliose Bangiales (Rhodophyta) in Iceland and the Faroe Islands. European Journal of Phycology 47(2): 146-159.

Mols-Mortensen A, CD Neefus, PM Pedersen \& J Brodie. 2014. Diversity and distribution of foliose Bangiales (Rhodophyta) in West Greenland: a link between the North Atlantic and North Pacific. European Journal of Phycology 49(1): 1-10.

Neefus CD, AC Mathieson, AS Klein, B Teasdale, T Gray \& C Yarish. 2002. Porphyra birdiae sp. nov. (Bangiales, Rhodophyta): a new species from the northwest Atlantic. Algae 17: 203-216.

Neefus CD, AC Mathieson, TL Bray \& C Yarish. 2008. The distribution, morphology, and ecology of three introduced asiatic species of Porphyra (Bangiales, Rhodophyta) in the Northwestern Atlantic. Journal of Phycology 44(6): 1399-1414.

Niwa K, H Furuita \& T Yamamoto. 2008a. Changes of growth characteristics and free amino acid content of cultivated Porphyra yezoensis Ueda (Bangiales Rhodophyta) blades with the progression of the number of harvests in a nori farm. Journal of Applied Phycology 20: 687-693.

Niwa K, A Kato, A Kobiyama, H Kawai \& Y Aruga. 2008b. Comparative study of wild and cultivated Porphyra yezoensis (Bangiales, Rhodophyta) based on molecular and morphological data. Journal of Applied Phycology 20(3): 261-270.

Niwa K, S Iida, A Kato, H Kawai, N Kikuchi, A Kobiyama \& Y Aruga. 2009. Genetic diversity and introgression in two cultivated species (Porphyra yezoensis and Porphyra tenera) and closely related wild species of Porphyra (Bangiales, Rhodophyta). Journal of Phycology 45(2): 493502.

Pollock DD \& WJ Bruno. 2000. Assessing an unknown evolutionary process: Effect of increasing site-specific knowledge through taxon addition. Molecular Biology and Evolution 17(12): 1854-1858.

R Development Core Team. 2014. R: a language and environment for statistical computing. R Foundation for Statistical Computing. <http://www.R-project.org/>

Ramírez ME. 1995. Recolección y colecciones científicas de macroalgas marinas. En: Alveal K, M Ferrario, E Oliveira \& E Sar (eds). Manual de métodos ficológicos, pp. 417-428. Universidad de Concepción, Concepción.
Ramírez ME \& B Santelices. 1991. Catálogo de las algas marinas bentónicas de la costa temperada del Pacífico de Sudamérica. Monografías Biológicas 5: 1-437. Facultad de Ciencias Biológicas, Pontificia Universidad Católica de Chile, Santiago.

Ramírez ME, L Contreras-Porcia, M-L Guillemin, J Brodie, C Valdivia, MR Flores-Molina, A Nuñez, C Bulboa \& C Lovazzano. 2014. Pyropia orbicularis sp. nov. (Rhodophyta, Bangiaceae) based on a population previously known as Porphyra columbina from the central coast of Chile. Phytotaxa 158(2): 133-153.

Saunders GW. 2005. Applying DNA barcoding to red macroalgae: a preliminary appraisal holds promise for future applications. Philosophical transactions of the Royal Society of London B: Biological Sciences 360(1462): 18791888.

Schonbeck MW \& TA Norton. 1979. The effects of brief periodic submergence on intertidal fucoid algae. Estuarine and Coastal Marine Science 8: 205-211.

Schonbeck MW \& TA Norton. 1980. Factors controlling the lower limits of fucoid algae on the shore. Journal of Experimental Marine Biology and Ecology 43: 131-150.

SERNAPESCA. 2016. Anuario estadístico de pesca. Servicio Nacional de Pesca, Valparaíso. <http://ww2.sernapesca.cl/ index.php?option=com_content\&view=article\&id=2303:anuarioestadistico-de-pesca-2016>

Sutherland JE, SC Lindstrom, WA Nelson, J Brodie, MDJ Lynch, MS Hwang, H-G Choi, M Miyata, N Kikuchi, MC Oliveira, T Farr, C Neefus, A Mols-Mortensen, D Milstein \& KM Müller. 2011. A new look at an ancient order: generic revision of the Bangiales (Rhodophyta). Journal of Phycology 47(5): 1131-1151.

Tamura K, D Peterson, N Peterson, G Stecher, M Nei \& S Kumar. 2011. MEGA5: MEGA5: molecular evolutionary genetics analysis using maximum likelihood, evolutionary distance, and maximum parsimony methods. Molecular Biology and Evolution 28(10): 2731-2739.

Underwood AJ \& P Jernakoff. 1984. The effects of tidal height, wave-exposure, seasonality and rock-pools on grazing and the distribution of intertidal macroalgae in New South Wales. Journal of Experimental Marine Biology and Ecology 75(1): 71-96.

Yarish C \& Pereira R. 2008. Mass production of marine macroalgae. Encyclopedia of Ecology 5: 2236-2247.

Recibido el 8 de agosto de 2016 y aceptado el 24 de abril de 2018

Editor: Claudia Bustos D. 